\title{
Endovascular Mechanical Thrombectomy for Acute Ischemic Stroke: A New Standard of Care
}

\author{
Dale Ding \\ Department of Neurosurgery, University of Virginia, Charlottesville, Virginia, USA
}

The treatment of acute ischemic stroke (AIS) in the setting of intracranial large artery occlusion (LAO) with intravenous tissue plasminogen activator (IV-tPA) is associated with low rates of recanalization and high rates of neurological morbidity and functional dependence. Endovascular intervention, particularly mechanical thrombectomy, is a promising therapeutic adjunct to IV-tPA for the treatment of acute LAO. However, until recently, its efficacy has been controversial. In this brief review, we analyze the criticisms of three negative randomized controlled trials (RCT) of endovascular stroke treatment and evaluate the results from seven positive endovascular stroke RCTs that have recently been presented or published. IMS III, MR RESCUE, and SYTHESIS Expansion were three RCTs that failed to show a benefit from endovascular stroke therapy. Major criticisms of these studies included a lack of routine screening for LAO, resulting in the selection of AIS patients without LAO for endovascular intervention, and a low utilization rate of modern endovascular thrombectomy devices, leading to substandard rates of successful recanalization. MR CLEAN was the first phase III RCT to show a significant clinical benefit from endovascular stroke therapy. The dissemination of its findings elicited a cascade of positive results from, to date, six additional endovascular stroke RCTs, ESCAPE, EXTEND-IA, SWIFT PRIME, REVASCAT, THERAPY, and THRACE, which were halted prematurely for efficacy. The cumulative evidence from these studies shows an overwhelming benefit from the endovascular treatment of acute LAO, therefore effectively establishing a new standard of care for the management of AIS.

Keywords Cerebral ischemia; Endovascular procedures; Revascularization; Reperfusion; Stroke; Stents
Correspondence: Dale Ding University of Virginia Department of Neurosurgery P.O. Box 800212 Charlottesville, VA 22908, USA

Tel: +1-434-924-2203

Fax: +1-434-982-5753

Email:dmd7q@hscmail.mcc.virginia.edu

Received: February 28, 2015

Revised: April 27, 2015

Accepted: April 28, 2015

The author has no financial conflicts of interest.

\section{Introduction}

Acute ischemic stroke (AIS) secondary to intracranial larger artery occlusion (LAO) is associated with significantly worse outcome than in the absence of LAO. Intravenous recombinant tissue plasminogen activator (IV-tPA) has been shown to be effective up to 4.5 hours from stroke onset, but recanalization rates for LAO are notoriously poor. Endovascular intervention has been studied as a potential adjunct to IV-tPA for patients with acute LAO, but, until recently, its efficacy has been unproven.
Thus, we aim to briefly review the critiques of the three negative endovascular stroke trials and analyze the results of seven recently completed trials of endovascular therapy for AIS.

\section{Criticisms of failed endovascular stroke trials}

IMS III, ${ }^{1}$ MR RESCUE, ${ }^{2}$ and SYNTHESIS Expansion ${ }^{3}$ were three multicenter, prospective, randomized controlled trials (RCT) which failed to show a benefit from endovascular inter- 
vention for AIS. Numerous concerns regarding various aspects of these trials were raised by the neurointerventional community. ${ }^{4}$ First, only one of the three RCTs, MR RESCUE, routinely identified LAO with either computed tomography or magnetic resonance angiography (CTA or MRA, respectively). In IMS III, CTA was performed in only $47 \%$ of patients, and approximately $20 \%$ of patients in the interventional arm either did not have a LAO or had an inaccessible, distally located thrombus. In SYNTHESIS Expansion, approximately $10 \%$ of patients in the interventional arm did not have a LAO.

Next, modern devices, such as retrievable stents (stentrievers) and the Penumbra System (Penumbra, Inc., Alameda, California, USA), were used in only a minority of patients. Stentrievers, such as the TREVO (Stryker Neurovascular, Fremont, California, USA) and Solitaire Flow Restoration (FR; ev3, Irvine, California, USA) devices, have been shown in RCTs, the TREVO 2 and SWIFT trials, respectively, to result in significantly better recanalization rates, faster reperfusion times, and better clinical outcomes compared to the first generation Merci retriever (Stryker Neurovascular) ${ }^{5,6}$ However, stentrievers or the Penumbra, alone or in combination, were used in only $22 \%, 39 \%$, and $19 \%$ of patients in the interventional arms of IMS III, MR RESCUE, and SYNTHESIS Expansion, respectively. The use of older, less effective endovascular technology resulted in significantly lower rates of successful recanalization, defined as Thrombolysis in Cerebral Ischemia (TICI) grade $2 \mathrm{~b}$ or 3 , than those reported in RCTs and prospective or retrospective registries using stentriever devices. The rates of TICI $2 \mathrm{~b}$ or 3 recanalization were $40 \%$ in IMS III, 27\% in MR RESCUE, and not reported in SYNTHESIS Expansion. Finally, patients in the interventional arm of SYNTHESIS Expansion were not administered IV-tPA, the use of which is supported by class I evidence, and were treated in a delayed fashion compared to the medical arm.

\section{Overview of successful endovascular stroke trials}

Despite the aforementioned criticisms, IMS III, MR RESCUE, and SYNTHESIS Expansion significantly dampened the initial enthusiasm surrounding endovascular therapy for AIS. The tide began to turn with a recently published RCT, MR CLEAN, which compared endovascular therapy to best medical management, with or without IV-tPA, within six hours of stroke onset. ${ }^{7} \mathrm{MR}$ CLEAN was the first phase III RCT to show a significant benefit from endovascular stroke therapy. Following the presentation the MR CLEAN results at the 2014 World Stroke Congress (Istanbul, Turkey), multiple ongoing endovascular stroke trials were halted for efficacy. The results from three RCTs, ESCAPE, ${ }^{8}$ EXTEND-
IA, ${ }^{9}$ and SWIFT PRIME, ${ }^{10}$ were initially presented at the 2015 International Stroke Congress (Nashville, Tennessee, USA), with the concurrent publication of ESCAPE and EXTEND-IA. Recently, the results from three additional RCTs, REVASCAT, ${ }^{11}$ THERAPY, ${ }^{12}$ and THRACE, ${ }^{13}$ were presented at the 2015 European Stroke Organization Conference (Glasgow, UK), with the concurrent publication of SWIFT PRIME and REVASCAT. The key findings of all seven endovascular stroke trials, from MR CLEAN onward, are summarized in Table 1.

MR CLEAN had the least restrictive inclusion criteria of the seven studies. ESCAPE, SWIFT PRIME, and REVASCAT used the Alberta stroke program early computed tomography score (ASPECTS) and EXTEND-IA used perfusion imaging to exclude patients with large core infarcts. THERAPY was the only study to use clot length to screen patients (minimum $8 \mathrm{~mm}$ for inclusion). The allowable time interval between stroke onset and intervention varied from 4.5 hours in THERAPY to 12 hours in ESCAPE. MR CLEAN, ESCAPE, and REVASCAT included patients outside of the 4.5-hours IV-tPA time window, whereas all patients in the control arms of EXTEND-IA, SWIFT PRIME, THERAPY, and THRACE received IV-tPA. Intervention was performed very rapidly after stroke onset in all RCTs, and stentriever devices were used in $82 \%$ and $86 \%$ of the interventional arms of MR CLEAN and ESCAPE, respectively, and in $100 \%$ of the interventional arms of EXTEND-IA, SWIFT PRIME, and REVASCAT. This resulted in significantly higher TICI $2 \mathrm{~b}$ or 3 recanalization rates in these studies compared to IMS III, MR RESCUE, and SYNTHESIS Expansion, although the rate of TICI $2 \mathrm{~b}$ or 3 recanalization in MR CLEAN (59\%) was relatively modest in the modern stentriever era.

All studies, with the exception of THERAPY, showed a significant improvement in the rate of functional independence (modified Rankin Scale score 0 to 2) at 90 days, with an absolute difference of $8 \%-31 \%$. THERAPY was halted before a significant benefit was observed in functional independence at 90 days $(P=0.52)$, but ordinal analysis showed significantly greater improvement in modified Rankin Scale for the interventional arm $(P=0.038)$. Although every study, except REVASCAT, reported a decrease in mortality with endovascular treatment, the difference was only statistically significant in ESCAPE (absolute difference, 8.6\%).

Some concerns were raised after presentation of the MR CLEAN results, regarding the modest rate of functional independence at 90 days (33\%, compared to $41 \%$ and $42 \%$ in the interventional arms of IMS III and SYNTHESIS Expansion, respectively) and the generalizability of a protocol limited to a single country (Netherlands) with a uniform healthcare system to a country like the United States, which has a much more heterogeneous healthcare environment. However, virtually any initial 


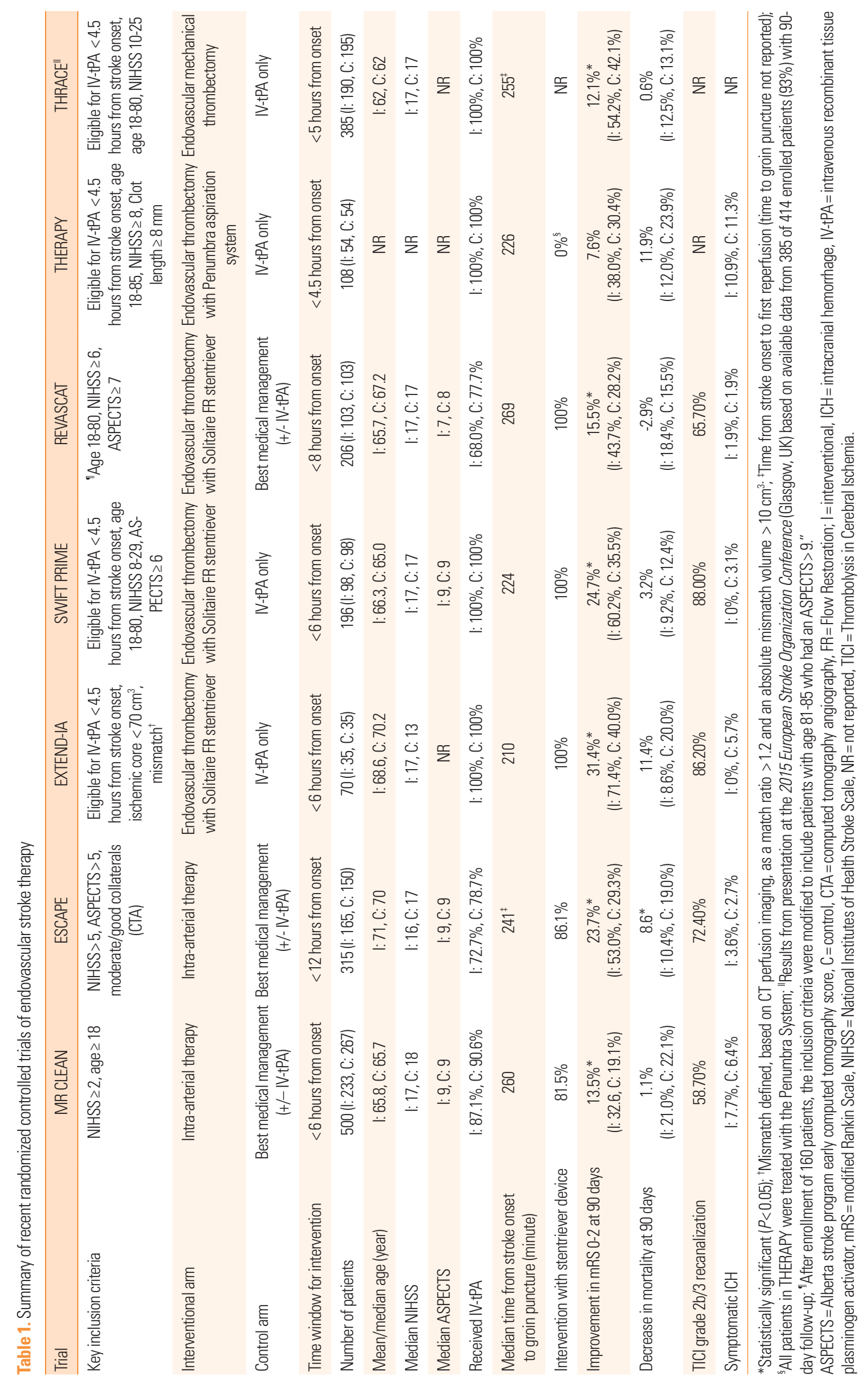


hesitation regarding the benefit of endovascular therapy for AIS from the MR CLEAN data has been quelled by concordant findings from the six subsequent RCTs. A wealth of additional information from these studies, including the detailed analyses of THERAPY and THRACE in their respective published manuscripts as well as pooled subgroup analyses, are forthcoming.

\section{Conclusions}

New evidence from MR CLEAN, ESCAPE, EXTEND-IA, SWIFT PRIME, REVASCAT, THERAPY, and THRACE has demonstrated an overwhelming benefit from endovascular intervention, preferably with stentriever-mediated mechanical thrombectomy, for the treatment of AIS secondary to LAO. Thus, a new standard of care for AIS management has been established. In order to provide our patients with the best available stroke care, cerebrovascular centers should seek to optimize workflow so that the appropriate patients can be rapidly imaged, selected for intervention, and triaged to the neurointerventional suite for endovascular therapy.

\section{References}

1. Broderick JP, Palesch YY, Demchuk AM, Yeatts SD, Khatri P, Hill MD, et al. Endovascular therapy after intravenous t-PA versus t-PA alone for stroke. N Engl J Med 2013;368:893-903.

2. Kidwell CS, Jahan R, Gornbein J, Alger JR, Nenov V, Ajani Z, et al. A trial of imaging selection and endovascular treatment for ischemic stroke. N Engl J Med 2013;368:914-923.

3. Ciccone A, Valvassori L, Nichelatti M, Sgoifo A, Ponzio M, Sterzi R, et al. Endovascular treatment for acute ischemic stroke. $N$ Engl J Med 2013;368:904-913.

4. Przybylowski CJ, Ding D, Starke RM, Durst CR, Crowley RW, Liu KC. Evolution of endovascular mechanical thrombectomy for acute ischemic stroke. World J Clin Cases 2014;2:614-622.

5. Nogueira RG, Lutsep HL, Gupta R, Jovin TG, Albers GW,
Walker GA, et al. Trevo versus Merci retrievers for thrombectomy revascularisation of large vessel occlusions in acute ischaemic stroke (TREVO 2): a randomised trial. Lancet 2012;380: 1231-1240.

6. Saver JL, Jahan R, Levy EI, Jovin TG, Baxter B, Nogueira RG, et al. Solitaire flow restoration device versus the Merci Retriever in patients with acute ischaemic stroke (SWIFT): a randomised, parallel-group, non-inferiority trial. Lancet 2012;380:1241-1249.

7. Berkhemer OA, Fransen PS, Beumer D, van den Berg LA, Lingsma HF, Yoo AJ, et al. A randomized trial of intraarterial treatment for acute ischemic stroke. N Engl J Med 2015;372: 11-20.

8. Goyal M, Demchuk AM, Menon BK, Eesa M, Rempel JL, Thornton J, et al. Randomized assessment of rapid endovascular treatment of ischemic stroke. $N$ Engl J Med 2015;372: 1019-1030.

9. Campbell BC, Mitchell PJ, Kleinig TJ, Dewey HM, Churilov L, Yassi N, et al. Endovascular therapy for ischemic stroke with perfusion-imaging selection. N Engl J Med 2015;372:1009-1018.

10. Saver JL, Goyal M, Bonafe A, Diener HC, Levy EI, Pereira $\mathrm{VM}$, et al. Stent-retriever thrombectomy after intravenous t-PA vs. t-PA alone in stroke. N Engl J Med 2015. doi: 10.1056/NEJMoa1415061.

11. Jovin TG, Chamorro A, Cobo E, de Miquel MA, Molina CA, Rovira A, et al. Thrombectomy within 8 hours after symptom onset in ischemic stroke. N Engl J Med 2015. doi: 10.1056/ NEJMoa1503780.

12. Mocco J, Khatri P, Zaidat O. The THERAPY trial: the randomized, concurrent controlled trial to assess the Penumbra System's safety and effectiveness in the treatment of acute stroke. European Stroke Organization Conference 2015. Glasgow, UK.

13. Bracard S, Investigators T. The contribution of intra-arterial thrombectomy in acute ischemic stroke in patients treated with intravenous thrombolysis. European Stroke Organization Conference 2015. Glasgow, UK. 